\title{
Human papillomavirus genotypes in anal intraepithelial neoplasia and anal carcinoma as detected in tissue biopsies
}

\author{
Anna K Wong ${ }^{1}$, Raymond C Chan ${ }^{1}$, Nidhi Aggarwal ${ }^{2}$, Manoj K Singh ${ }^{3}$, W Stephen Nichols ${ }^{1}$ \\ and Shikha Bose ${ }^{1}$ \\ ${ }^{1}$ Department of Pathology and Laboratory Medicine, and the George Burns and Gracie Allen Research \\ Institute, Cedars-Sinai Medical Center, Los Angeles, CA, USA ${ }^{2}$ Department of Pathology, University of \\ Pittsburgh Medical Center, Pittsburgh, PA, USA and ${ }^{3}$ Department of Pathology, All India Institute of Medical \\ Sciences, New Delhi, India
}

\begin{abstract}
Human papillomavirus (HPV) infection strongly correlates with the development of anal intraepithelial neoplasias and carcinomas; however, few studies have characterized the distribution of the specific subtypes of the virus in the varying grades of dysplasia. This report characterizes the distribution of HPV 16/18 in surgical specimens with anal intraepithelial neoplasia (AIN) I-III and histological variants of anal carcinoma. A total of 111 anal surgical specimens with no dysplasia (10), AIN I-III (53), and anal carcinomas (48) were evaluated for the presence of high-risk HPV infection and subtyped by nested PCR or the Invader Assay. High-risk virus types were detected in progressively greater number of anal intraepithelial lesions from $56 \%$ in low grade to $88 \%$ in high grade. Type 16 was the prevalent subtype and was noted in $28 \%$ of low grade and $68 \%$ of high-grade lesions. Moderate dysplasias showed type 16 in $20 \%$, a prevalence similar to that in low-grade lesions. The non$16 / 18$ subtypes of the virus predominated and were present in $50 \%$ of the cases. Most $(89 \%)$ squamous carcinomas were associated with high-risk viruses, $68 \%$ with type 16 , a prevalence similar to that noted in highgrade dysplasia. Non-16/18 subtypes were encountered more frequently in squamous carcinomas from immunodeficient individuals ( $57 \%$ cases) as compared with immunocompetent individuals $(18 \%$ cases). The similarity in the prevalence of type 16 in high-grade dysplasia and squamous carcinomas suggests that anal intraepithelial lesion III is the true precursor of squamous carcinoma and warrants aggressive management. Anal intraepithelial lesions II showed a virus distribution that was similar to low-grade dysplasia. In addition, a subset of these that were associated with type 16 or 18 showed progression, whereas those associated with non-16/18 subtypes regressed, thereby raising the possibility of conservative management for these lesions. Modern Pathology (2010) 23, 144-150; doi:10.1038/modpathol.2009.143; published online 16 October 2009
\end{abstract}

Keywords: anal intraepithelial neoplasia; anal carcinoma; anal dysplasia; human papillomavirus 16; human papillomavirus 18

The human papillomavirus (HPV) has been implicated as a significant factor in anal carcinogenesis. Eighty-four percent of anal cancers are associated with oncogenic/high-risk strains of HPV with type 16 being the most prevalent strain accounting

Correspondence: Dr S Bose, MD, Department of Pathology and Laboratory Medicine, and the George Burns and Gracie Allen Research Institute, Room \#8732, Cedars-Sinai Medical Center, 8700 Beverly Boulevard, Los Angeles, CA 90048, USA.

E-mail: Shikha.bose@cshs.org

Received 5 May 2009; revised 23 June 2009; accepted 26 June 2009; published online 16 October 2009 for $70 \%$ of anal carcinomas. ${ }^{1-3}$ Anal carcinoma was a relatively uncommon malignancy with an incidence of 0.7 per 100000 men and 0.9 per 100000 women. ${ }^{4}$ Since the onset of AIDS, its incidence has risen by $96 \%$ in men and 39\% in women and continues to do so yearly reaching epidemic proportions in HIV-infected males who have sex with males and is attributed to the improved life span because of antiretroviral therapy. ${ }^{4}$ Aside from HIV, anal carcinoma has also been observed in transplant recipients who have a 10-fold excess risk of anal cancer as compared with the normal population. ${ }^{4}$ 
Similar to the cervix, anal carcinomas are associated with anal intraepithelial neoplasia (AIN) and high-grade AIN is believed to be the precursor lesion to anal carcinomas. Low-grade AIN lesions have a variable course progressing to high-grade AIN in $52-66 \%$ of HIV positive individuals and in $17 \%$ of HIV negative individuals. ${ }^{5}$ This difference is attributed to immunodeficiency that fosters the persistence of the virus leading to progression of disease. In HIV-infected men, anal HPV is detected in $>90 \%$ of cases and infections with multiple HPV types are common. ${ }^{6}$ Although several studies have evaluated the presence of HPV in anal cytology specimens, few studies have examined the prevalence of HPV types in the different grades of AIN and subtypes of anal carcinomas in tissue sections. Studies in tissue sections are necessary for determining the specific HPV types in specific histopathologic lesions. In a comparative study between concurrent anal cytology swabs and tissue biopsies obtained at the same visit, Gohy et $a l^{7}$ found poor correlation between positivity for HPV DNA in the two modalities. Cytology specimens showed a higher frequency of HPV detection and greater burden of infection because of its ability to sample the entire anal canal surface as opposed to anal biopsies, which assess only specific lesions. Thus, analysis of anal cytology for HPV may overestimate the type-specific associations for dysplasia. The function of specific HPV types as it relates to the histologic grade of dysplasia, the natural history, and malignant potential has not been extensively studied; thus, the development of personalized treatment guidelines have been limited. This study was designed to determine the HPV types involved in the different grades of AIN and different subtypes of anal carcinoma in tissue specimens from immunodeficient and immunocompetent individuals.

\section{Materials and methods}

\section{Specimen Selection}

One hundred and eleven anal surgical biopsy and resection specimens were retrieved from our files between 2003 and 2007. This included 10 cases negative for dysplasia, 18 AIN I, 10 AIN II, 25 AIN III, 29 squamous cell carcinoma not otherwise specified, 9 basaloid squamous cell carcinoma, and 10 adenocarcinomas. Twenty-seven specimens were from females, whereas 84 were from males. Their ages ranged from 19 to 93 years (mean 50 years). Thirty-nine (35\%) patients were immunodeficient, $35(90 \%)$ of which were HIV positive and 4 were immunosuppressed from medications taken for inflammatory bowel disease or orthotopic renal transplant. Twenty-four patients were HIV negative. Forty-eight patients were not tested for HIV and were presumed to be negative.

Histology from all cases was reviewed by two pathologists who confirmed the diagnosis.
Discrepant cases were resolved by histologic evaluation by a third pathologist. Representative blocks of the lesions were selected for HPV testing. Charts were reviewed for clinical follow-up for all patients diagnosed with AIN.

\section{HPV Testing}

DNA was extracted from each of the 111 formalinfixed and paraffin-embedded specimens using the QIAamp DNA Mini kit as per the manufacturer's recommendations. Five $10 \mu$ thick sections were lysed by the QIAGEN Proteinase $\mathrm{K}$ in the presence of the lysis buffer. Total DNA was then extracted by ethanol (96-100\%), purified using QIAamp Mini spin columns in a standard microcentrifuge and washed after adsorption onto the QIAamp silica membrane to remove protein and other contaminants. Purified DNA was then eluted from the QIAamp Mini spin column and used for detecting the presence of high-risk HPV.

High-risk HPV was detected by nested PCR in 48 cases of anal carcinoma and by the Invader Screening Assay in 10 normal cases and 52 cases of AIN. One case was tested by both methodologies to confirm similar results. All specimens were additionally tested for HPV 16 and 18 by PCR (anal carcinomas) or the Invader Type-Specific HPV Assays for HPV 16 and 18 (normal biopsies and AIN).

\section{Nested PCR}

Nested PCR was performed using primers for MY09/ 11 and GP05/06 with the following sequences: MY09: CGT CCM ARR GGA WAC TGA TC; MY11: GCM CAG GGW CAT AAY AAT GG; G5: TTT GTT ACT GTG GTA GAT ACT AC, and G6: GAA AAA TAA ACT GTA AAT CAT ATT C. ${ }^{8}$ After this, typespecific testing for HPV 16 and 18 was performed (primers F96/R96: GGT CGG TGG ACC GGT CGA TG and GCA ATG TAG GTG TAT CTC CA for HPV 16 and primers F115/R115: CCT TGG ACG TAA ATT TTT GG and CAC GCA CAC GCT TGG CAG GT for HPV 18). ${ }^{9}$ Beta-globin was coamplified in all reactions to determine specimen adequacy.

\section{Invader Assay}

Extracted DNA was tested for the presence of highrisk HPV and HPV 16 and 18 by Invader (Hologic, Inc., Bedford, MA, USA), as described by us earlier in accordance with the manufacturer's recommendations. $^{10}$

Fluorescent In Situ Hybridization for HR-HPV DNA A measure of $5 \mu \mathrm{m}$ micron thick sections of the two adenocarcinomas that tested positive for HR HPV using PCR were subjected to in situ hybridization using the Dako GenPoint High-Risk HPV Biotinylated DNA Probe Cocktail (HPV 16, 18, 31, 33, 35, $39,45,51,52,56,58,59,68)$ and the GenPoint 
Tyramide Signal Amplification System (Dako Corp., Carpinteria, CA, USA) as per the manufacturer's recommendations.

\section{Results}

\section{High-Risk HPV in AIN}

Overall high-risk HPV was detected in 40 of 53 $(75 \%)$ cases with AIN of which $24(60 \%)$ were associated with HPV 16, 3 (7.5\%) with HPV 18, and $14(26 \%)$ with non-16/18 high-risk HPV (Table 1). AIN from 20 of the $29(69 \%)$ immunodeficient patients were high-risk HPV positive as compared with 20 of the 24 (83\%) immunocompetent patients. HPV 16 comprised a large proportion of the highrisk HPV (immunodeficient 13/20, 65\%; immunocompetent $11 / 20,55 \%$ ) in both groups. Non-16/18 high-risk HPV were detected in 6/20 AIN from immunodeficient and 8/20 immunocompetent individuals and comprised 30 and $40 \%$, respectively, of the high-risk HPV types.

Ten $(56 \%)$ of the 18 AIN I cases were positive for high-risk HPV, 5 (50\%) of which were HPV 16, 1 $(10 \%)$ HPV 18 and the remaining 4 (40\%) were non16/18 high-risk HPV. Nine cases of AIN I were from immunodeficient and nine from immunocompetent individuals. AIN I lesions from immunocompetent individuals were more often high-risk HPV positive as compared with those from immunodeficient patients $(6 / 9,67$ vs 4/9, 44\%). In both groups, HPV 16 comprised $50 \%$ of the high-risk HPV.

Of the 10 patients with AIN II, $8(80 \%)$ were highrisk HPV positive of which the majority $(5,63 \%)$ were non-16/18 high-risk HPV, 2 (25\%) were HPV 16, and $1(13 \%)$ HPV 18. Seven of the AIN II lesions were from immunodeficient and three from immunocompetent individuals. High-risk HPV positivity was noted in all $(3 / 3,100 \%)$ of the immunocompe- tent individuals and in five $(71 \%)$ of the immunodeficient patients. In both of these groups, non-16/ 18 high-risk HPV types predominated.

Twenty two $(88 \%)$ of the 25 AIN III cases were positive for high-risk HPV including 17 (68\%) with HPV 16, 1 (4\%) patient with HPV 18, and $5(20 \%)$ with non-16/18 high-risk HPV. One patient contained DNA of both HPV 16 and 18. Of the 25 patients with AIN III, 13 were immunodeficient and 12 immunocompetent. Eleven patients from each of the two groups (immunodeficient $85 \%$ and immunocompetent $92 \%$ ) were high-risk HPV positive. HPV 16 comprised the dominant type in both of these groups (immunodeficient 9, 69\% and immunocompetent $8,67 \%$ ).

All normal specimens were from immunocompetent individuals and were HPV negative.

\section{High-Risk HPV in Anal Carcinomas}

Thirty four (89\%) of all the squamous cell carcinoma were high-risk HPV positive, 26 (76\%) of which were positive for HPV 16, 1 (3\%) for HPV 18, and 8 $(24 \%)$ for non-16/18 high-risk HPV (Table 2). Nine of the cancers were from immunodeficient patients and all tested positive for high-risk HPV, 5 (56\%) of which were HPV 16, and the remaining $4(44 \%)$ were non-16/18 high-risk HPV. The remaining 29 cancers were from immunocompetent individuals, $25(85 \%)$ squamous cell carcinoma tested positive for high-risk HPV. Of these, the majority $(21,84 \%)$ was HPV 16, 4 (16\%) were non-16/18 high-risk HPV, and $1(4 \%)$ was HPV 18. One cancer with HPV 18 was also tested positive for HPV 16.

High-risk HPV was detected in 26 (90\%) of the 29 squamous cell carcinoma not otherwise specified of which 18 (70\%) were HPV 16, 1 (4\%) HPV 18, and 8 $(31 \%)$ non-16/18 high-risk HPV. One case showed

Table 1 Prevalence of high-risk HPV in anal dysplasia

\begin{tabular}{|c|c|c|c|c|c|}
\hline Diagnosis & Number & $\begin{array}{c}\text { High-risk } \\
\text { HPV+ }\end{array}$ & $H P V 16+$ & HPV 18+ & $\begin{array}{l}\text { Non-HPV 16/18 } \\
\text { high-risk HPV+ }\end{array}$ \\
\hline Normal & 10 & 0 & 0 & 0 & 0 \\
\hline Immunocompetent & 10 & 0 & 0 & 0 & 0 \\
\hline AIN-all & 53 & $40(75 \%)$ & $24(45 \%)^{a}$ & $3(6 \%)^{a}$ & $14(26 \%)$ \\
\hline Immunodeficient & 29 & $20(69 \%)$ & $13(45 \%)^{\mathrm{a}}$ & $2(6 \%)^{a}$ & $6(21 \%)$ \\
\hline Immunocompetent & 24 & $20(83 \%)$ & $11(46 \%)$ & $1(4 \%)$ & $8(33 \%)$ \\
\hline AIN I & 18 & $10(56 \%)$ & $5(28 \%)$ & $1(6 \%)$ & $4(22 \%)$ \\
\hline Immunodeficient & 9 & $4(44 \%)$ & $2(22 \%)$ & $1(11 \%)$ & $1(11 \%)$ \\
\hline Immunocompetent & 9 & $6(67 \%)$ & $3(33 \%)$ & 0 & $3(33 \%)$ \\
\hline AIN II & 10 & $8(80 \%)$ & $2(20 \%)$ & $1(10 \%)$ & $5(50 \%)$ \\
\hline Immunodeficient & 7 & $5(71 \%)$ & $2(29 \%)$ & 0 & $3(43 \%)$ \\
\hline Immunocompetent & 3 & $3(100 \%)$ & 0 & $1(33 \%)$ & $2(67 \%)$ \\
\hline AIN III & 25 & $22(88 \%)$ & $17(68 \%)^{\mathrm{a}}$ & $1(4 \%)^{a}$ & $5(20 \%)$ \\
\hline Immunodeficient & 13 & $11(85 \%)$ & $9(69 \%)^{\mathrm{a}}$ & $1(8 \%)^{a}$ & $2(15 \%)$ \\
\hline Immunocompetent & 12 & $11(92 \%)$ & $8(67 \%)$ & 0 & $3(25 \%)$ \\
\hline
\end{tabular}

${ }^{\mathrm{a}}$ Includes a case each of AIN III with both HPV 16 and 18.

AIN, anal intraepithelial neoplasia. 
both HPV 16 and 18. Seven of the cases were from immunodeficient individuals, all showed high-risk HPV; however, only 3 (43\%) were HPV 16, whereas the remaining $4(57 \%)$ were non-16/18 high-risk HPV. In contrast, in the 22 immunocompetent patients with squamous cell carcinoma not otherwise specified, 19 (86\%) were high-risk HPV positive of which the majority $(15,79 \%)$ were HPV 16, $4(21 \%)$ were non-16/18 high-risk HPV, and 1 (5\%) HPV 18.

Of the nine basaloid squamous cell carcinoma, high-risk HPV was detected in eight $(89 \%)$, all of which were HPV 16. Two of the cancers were from immunodeficient patients, both showed HPV 16. The remaining seven patients were immunocompetent, six $(86 \%)$ of their cancers were high-risk HPV and HPV 16 positive.

Only 2 of the 10 adenocarcinomas (20\%) were positive for high-risk HPV, one of which showed a weak band on PCR (low levels of virus). One patient was immunodeficient and the remaining nine were immunocompetent. High-risk HPV non-16/18 type was detected in the cancer from the immunodeficient patient, whereas the cancer from the immunocompetent individual showed low levels of HPV 16. High-risk HPV, however, was not detectable by fluorescent in situ hybridization in both of the cases.

Overall, high-risk HPV was present in progressively greater numbers of AIN lesions from AIN I $(56 \%)$ to AIN III (88\%). HPV 16 was the prevalent subtype accounting for half of the high-risk HPV overall. However, a difference was noted in its prevalence in the different grades of AIN. AIN I showed HPV 16 in $28 \%$ of cases, whereas in AIN III, HPV 16 was detected in $68 \%$ of the samples. AIN II lesions showed HPV 16 in 20\%, a prevalence similar to that in AIN I. A majority of AIN II (50\%) was associated with the non-16/18 subtypes of high-risk HPV. Anal squamous cell carcinoma was associated with high-risk HPV in 89\% and with HPV 16 in $68 \%$, similar to that noted in AIN III. High-risk HPV was associated with most squamous cell carcinoma irrespective of immune status: $100 \%$ in squamous cell carcinoma from immunodeficient individuals and $86 \%$ in immunocompetent individuals. HPV 16 was the dominant virus type noted in both groups regardless of immune system status comprising 56$72 \%$ of cases. Non-16/18 subtypes were encountered more frequently in squamous cell carcinoma not otherwise specified from immunodeficient individuals ( $57 \%$ cases) as compared with immunocompetent individuals (18\% cases); however, this difference did not reach statistical significance. Basaloid squamous cell carcinomas were all HPV 16 positive. Adenocarcinomas were rarely associated with high-risk HPV.

\section{Clinical Course}

Follow-up information was available in 26 patients diagnosed with AIN I-AIN III with follow-up periods ranging from 1 to 29 months (median: 13 months). Twelve patients had a subsequent anal biopsy and 15 patients anal pap smears. Of the four patients with AIN I, three (two high-risk HPV negative and one non-16/18 high-risk HPV type) showed persistent AIN I at 3-16 months (Table 3). All were immunocompetent. One patient, immunodeficient with HPV 18, showed AIN III on follow-up biopsy 1 month later. Six patients with AIN II had follow-up. Five showed less severe lesions at 4-21 months. All were immunocompetent, two were high-risk HPV negative, two were high-risk HPV non-16/18 type, and one was HPV 18 positive. One immunodeficient patient with AIN II and HPV 16 showed AIN III at 7 months. Of the 25 AIN III patients, follow-up was available in 17. One immunodeficient patient with both HPV 16 and 18 progressed to squamous cell carcinoma at 12 months. Six patients showed persistent HSIL lesions at 2-29 months, half ${ }^{3}$ of whom were immunocompetent and half ${ }^{3}$ immunodeficient. Three were HPV 16 positive, two were non-16/18 types, and one was

Table 2 Prevalence of high-risk HPV in anal carcinoma

\begin{tabular}{|c|c|c|c|c|c|}
\hline Diagnosis & Number & $\begin{array}{c}\text { High-risk } \\
\text { HPV+ }\end{array}$ & $H P V 16+$ & HPV 18+ & $\begin{array}{l}\text { Non-HPV 16/18 } \\
\text { high-risk HPV+ }\end{array}$ \\
\hline Squamous cell carcinoma-all & 38 & $34(89 \%)$ & $26(68 \%)^{\mathrm{a}}$ & $1(3 \%)^{\mathrm{a}}$ & $8(21 \%)$ \\
\hline Immunodeficient & 9 & $9(100 \%)$ & $5(56 \%)$ & 0 & $4(44 \%)$ \\
\hline Immunocompetent & 29 & $25(86 \%)$ & $21(72 \%)^{\mathrm{a}}$ & $1(3 \%)^{\mathrm{a}}$ & $4(14 \%)$ \\
\hline Squamous cell carcinoma-not otherwise specified & 29 & $26(90 \%)$ & $18(62 \%)^{\mathrm{a}}$ & $1(3 \%)^{\mathrm{a}}$ & $8(28 \%)^{a}$ \\
\hline Immunodeficient & 7 & $7(100 \%)$ & $3(43 \%)$ & 0 & $4(57 \%)$ \\
\hline Immunocompetent & 22 & $19(86 \%)$ & $15(68 \%)^{\mathrm{a}}$ & $1(5 \%)^{\mathrm{a}}$ & $4(18 \%)^{\mathrm{a}}$ \\
\hline Squamous cell carcinoma- basaloid & 9 & $8(89 \%)$ & $8(89 \%)$ & 0 & 0 \\
\hline Immunodeficient & 2 & $2(100 \%)$ & $2(100 \%)$ & 0 & 0 \\
\hline Immunocompetent & 7 & $6(86 \%)$ & $6(86 \%)$ & 0 & 0 \\
\hline Adenocarcinoma & 10 & $2(20 \%)$ & $1(10 \%)$ & 0 & $1(10 \%)$ \\
\hline Immunodeficient & 1 & 1 & 0 & 0 & 1 \\
\hline Immunocompetent & 9 & $1(11 \%)$ & $1(11 \%)$ & 0 & 0 \\
\hline
\end{tabular}

ancludes a case of squamous cell carcinoma infected with both HPV 16 and 18. 
Table 3 Clinical course of patients with anal intraepithelial neoplasia (AIN)

\begin{tabular}{|c|c|c|c|c|c|c|}
\hline $\begin{array}{l}\text { Follow up } \\
\text { anal biopsy (n) }\end{array}$ & $\begin{array}{l}\text { Initial } \\
\text { diagnosis }\end{array}$ & Follow-up diagnosis & Cases & $\begin{array}{l}\text { High-risk HPV } \\
\text { type (n) }\end{array}$ & Immune status & $\begin{array}{l}\text { Follow-up } \\
\text { (months) }\end{array}$ \\
\hline \multirow[t]{3}{*}{ More severe (3) } & AIN I & AIN III & 1 & 18 & Immunodeficient & 1 \\
\hline & AIN II & AIN III & 1 & 16 & Immunodeficient & 7 \\
\hline & AINIII & Squamous cell carcinoma & 1 & 16 and 18 & Immunodeficient & 12 \\
\hline \multirow[t]{2}{*}{ Same (9) } & AINI & AIN I & 3 & $\begin{array}{l}\text { Non-16/18 (1) } \\
\text { Negative (2) }\end{array}$ & Immunocompetent & $3-16$ \\
\hline & AIN III & HSIL/AIN III & 6 & $\begin{array}{l}16(3) \\
\text { Non-16/18 (2) } \\
\text { Negative (1) }\end{array}$ & $\begin{array}{l}\text { Immunocompetent (3) } \\
\text { Immunodeficient (3) }\end{array}$ & $2-29$ \\
\hline \multirow[t]{2}{*}{ Less severe (14) } & AIN II & $\begin{array}{l}\text { Negative (1) } \\
\text { ASC/LSIL (4) }\end{array}$ & 5 & $\begin{array}{l}18(1) \\
\text { Non-16/18 (2) } \\
\text { Negative (2) }\end{array}$ & Immunocompetent & $4-21$ \\
\hline & AIN III & $\begin{array}{l}\text { Negative (3) } \\
\text { ASC/LSIL (6) }\end{array}$ & 9 & $\begin{array}{l}16(8) \\
\text { Non-16/18 (1) }\end{array}$ & $\begin{array}{l}\text { Immunocompetent (5) } \\
\text { Immunodeficient (4) }\end{array}$ & $2-26$ \\
\hline
\end{tabular}

ASC, atypical squamous cells; LSIL, low-grade squamous intraepithelial lesion; HSIL, high-grade squamous intraepithelial lesion.

high-risk HPV negative. Nine AIN III showed lowgrade abnormalities on follow-up at 2-26 months, five were immunocompetent, and four were immunodeficient. The majority ${ }^{8}$ were associated with HPV 16, whereas one was of the non-16/18 type.

To summarize, overall three cases showed progression to more severe lesions on follow-up. All were immunodeficient and were associated with either HPV 16 or 18. Nine cases showed persistent lesions, the majority were immunocompetent (6/9) with variable HPV status. Fourteen cases showed less severe lesions on follow-up. The majority were immunocompetent (10/14) with variable HPV types.

\section{Discussion}

Our understanding of AIN and anal carcinoma remains limited despite its increasing incidence within the last 25 years; thus, there is an urgent need for understanding the natural history and the function of HPV in anal carcinogenesis. ${ }^{11}$ Anal carcinoma and AIN have both been linked to HPV infection, ${ }^{1,2,12,13}$ of which over 100 genotypes have been identified. At least 40 subtypes are known to infect the anogenital mucosa with variable neoplastic risk. High-risk subtypes (HPV 16, 18, 31, 33, 35, $39,45,51,52,56,58,59,66,69)$ are associated with $84 \%$ of high-grade intraepithelial lesions. ${ }^{14}$ Nonsquamous malignancies of the anal canal, adenocarcinomas of the rectum, and benign anal mucosa have all tested negative for HPV., ${ }^{3,15-17}$ Our findings in this study are concordant with the literature. High-risk HPV was present in $89 \%$ of squamous cell carcinoma overall, $100 \%$ of cancers from immunodeficient individuals, and $86 \%$ of cancers in immunocompetent individuals.

HPV 16 has been identified as the major oncogenic strain contributing to $56-73 \%$ of anal cancers. ${ }^{13,17}$ We found HPV 16 in $68 \%$ of all squamous cell carcinoma with some minor differences in the different subtypes. Although most studies have reported similar observation, ${ }^{1,18,19}$ others have noted no difference. ${ }^{19,20}$ Non-basaloid squamous cell carcinoma showed a lower prevalence of HPV 16 (62\%) as compared with basaloid squamous cell carcinoma (89\%). In addition, a proportion (21\%) of nonbasaloid squamous cell carcinoma was involved by high-risk HPV that was not HPV 16. On the other hand, all basaloid squamous cell carcinoma contained HPV 16. Thus, although traditional squamous cell carcinoma and basaloid squamous cell carcinoma show a similar proportion of high-risk HPV, HPV 16 seems to have a greater function in carcinomas with basaloid differentiation.

HPV 18 was infrequently identified and was detected in only $3 \%$ of the squamous cell carcinoma, a finding that has been observed earlier by Frisch et $a{ }^{1}{ }^{1}$ who noted HPV 18 in only $6 \%$ of the 388 cancers examined. Although HPV 18 is the second most critical strain in the cervix, we found it in only 1 of $38(3 \%)$ squamous cell carcinomas-a case co-infected with HPV 16. In the cervix, HPV 18 has been commonly associated with adenocarcinomas. ${ }^{20} \mathrm{HPV} 18$ was not detected in any of our patients with anal adenocarcinomas; however, highrisk HPV was observed in two of the cases by PCR, one of which showed low levels of HPV 16. The presence of the high-risk HPV, however, could not be confirmed by fluorescent in situ hybridization studies on these two cases. Although fluorescent in situ hybridization is known to be a less sensitive test, and a negative result does not rule out the presence of HPV, the additional possibility of contamination from an insipient concurrent infection in adjacent normal tissue resulting in the positive PCR cannot be excluded.

An interesting observation from our study was the greater prevalence of the non-16/18 genotypes in anal carcinomas from immunodeficient individuals as compared with immunocompetent persons (44 vs $14 \%$, respectively), although this difference did not reach statistical significance. This finding thus far has not been reported and additional studies are 
warranted to confirm the function of non-16/18 high-risk HPV subtypes in anal carcinomas of immunodeficient individuals.

AIN is believed to be the precursor lesion of anal carcinoma and is found in both HIV positive and negative individuals with a greater prevalence in immunodeficient individuals. Various studies report a prevalence of $36-52 \%$ in HIV positive individuals and $8-17 \%$ in HIV negative men and women. ${ }^{21,22}$ Approximately, a third of our patients with anal biopsies or resections were immunodeficient with an overwhelming majority (90\%) being infected with HIV. Although AIN occurs with greater frequency in immunodeficient individuals, AIN lesions from the immunodeficient individuals in our series did not show a greater prevalence of highrisk HPV. We noted high-risk HPV in 69\% of AIN from immunodeficient patients as compared with $83 \%$ from immunocompetent individuals. Therefore, the increased incidence of AIN in immunodeficient individuals seems to be related to the greater persistence of the virus in these patients. Several studies have reported a two to six times greater prevalence of HPV infection in immunosuppressed patients ${ }^{23-28}$ and twice the risk of progression from low-grade AIN to high-grade AIN. ${ }^{27-30}$ AIN lesions are known to advance from low-grade to high-grade AIN within 2 years of diagnosis in $36-66 \%$ of individuals with progression occurring more rapidly and more frequently in immunodeficient individuals. ${ }^{27,30}$

Our study confirms the increasing prevalence of high-risk HPV with worsening dysplasia: 56\% of AIN I, $80 \%$ of AIN II, and $88 \%$ of AIN III, findings earlier reported by Varnai et $a l^{31}$ who noted the frequency of high-risk HPV increased from $0 \%$ in AIN I to $33 \%$ in AIN II and $94 \%$ in AIN III.

A similar trend was noted with prevalence of HPV $16,28 \%$ of AIN I, $20 \%$ of AIN II, and $68 \%$ of AIN III comparable with Varnai et $a l^{31}$ who found no cases of HPV 16 in AIN I, 2/7 (29\%) AIN II, and 15/18 $(83 \%)$ of AIN III and Gohy et $a l^{7}$ who detected HPV 16 in $10 \%$ of AIN I and $36 \%$ of AIN II/III cases. The prevalence of HPV 16 in AIN III is similar to that noted in squamous cell carcinoma, whereas its prevalence in AIN I and AIN II were similar, raising the possibility that AIN II lesions could potentially behave similar to AIN I lesions. An analogous situation has recently been reported in gynecologic literature. Castle et $a 1^{32}$ in a sub-analysis of the ALTS data reported that $40 \%$ of CIN II cases would regress if left untreated. They also noted that CIN II caused by HPV 16 may be less likely to regress than CIN II caused by other high-risk HPV types. ${ }^{32}$ In our study, six $(60 \%)$ of AIN II lesions were non-16 high-risk HPV types; five of which on follow-up showed less severe lesions. One AIN II with HPV 16 had followup and progressed to AIN III. This raises the possibility that AIN II lesions containing non-16 HPV types could potentially be treated conservatively. Several specialists favor a conservative approach to the management of anal dysplasia because of the significantly higher rate of recurrence known to occur in individuals with anal dysplasias, particularly in the immunodeficient. ${ }^{33}$ Moreover, surgical treatment is not as effective in eradicating disease as in the cervix and may additionally be limited by impaired healing in the immunodeficient individuals. ${ }^{4}$ Thus, subtyping for HPV 16 and 18 may offer a means of selecting individuals with AIN II for aggressive vs conservative management.

In summary, our study shows that HPV 16 is the prevalent HPV subtype and is associated with all grades of AIN and anal squamous carcinomas. AIN III seems to be the true precursor lesion of squamous cell carcinoma and warrants aggressive management. AIN I and II lesions showed similar HPV subtype associations. AIN II lesions associated with HPV 16 and/or 18 showed progression, whereas those associated with non-HPV 16/18 subtypes regressed, thereby raising the possible development of defined surveillance strategies and tailored management protocols for these two groups based on HPV subtypes.

\section{Disclosure/conflict of interest}

The authors declare no conflict of interest.

\section{References}

1 Frisch M, Fenger C, van den Brule AJ, et al. Variants of squamous cell carcinoma of the anal canal and perianal skin and their relation to human papillomaviruses. Cancer Res 1999;59:753-757.

2 Melbye M, Palefsky J, Gonzales J, et al. Immune status as a determinant of human papillomavirus detection and its association with anal epithelial abnormalities. Int J Cancer 1990;46:203-206.

3 Zbar AP, Fenger C, Efron J, et al. The pathology and molecular biology of anal intraepithelial neoplasia: comparisons with cervical and vulvar intra-epithelial carcinoma. Int J Colorectal Dis 2002;17:203-215.

4 Abbasakoor F, Boulos PB. Anal intraepithelial neoplasia. Br J Surg 2005;92:277-290.

5 Lacey HB, Wilson GE, Tilston P, et al. A study of anal intraepithelial neoplasia in HIV positive homosexual men. Sex Transm Infect 1999;75:172-177.

6 Palefsky JM, Holly EA, Efirdc JT, et al. Anal intraepithelial neoplasia in the highly active antiretroviral therapy era among HIV-positive men who have sex with men. AIDS 2005;19:1407-1414.

7 Gohy L, Gorska I, Rouleau D, et al. Genotyping of human papillomavirus DNA in anal biopsies and anal swabs collected from HIV-seropositive men with anal dysplasia. J Acquir Immune Defic Syndr 2008;49: 32-39.

8 Sotlar K, Diemer D, Dethleffs A, et al. Detection and typing of human papillomavirus by E6 nested multiplex PCR. J Clin Microbiol 2004;42:3174-3184.

9 Bidermann K, Dandachi N, Trattner $\mathrm{M}$, et al. Comparison of real-time PCR signal-amplified in situ hybridization and conventional PCR for detection and 
quantification of human papillomavirus in archival cervical cancer tissue. J Clin Microbiol 2004;42: 3758-3765.

10 Wong AK, Chan RC, Nichols WS, et al. Human papillomavirus (HPV) in atypical squamous cervical cytology: the Invader HPV tests as a new screening assay. J Clin Microbiol 2008;46:869-875.

11 Uronis HE, Bendell JC. Anal cancer: an overview. Oncologist 2007;12:524-534.

12 Frazer IH, Medley G, Crapper RM, et al. Association between anorectal dysplasia, human papillomavirus, and human immunodeficiency virus infection in homosexual men. Lancet 1986;2:657-660.

13 Frisch M, Glimelius B, van den Brule AJC, et al. Sexually transmitted infection as a cause of anal cancer. N Engl J Med 1997;337:1350-1358.

14 Papaconstantinou HT, Lee AJ, Simmang CL, et al. Screening methods for high-grade dysplasia in patients with anal condyloma. J Surg Res 2005;127:8-13.

15 Friedman HB, Saah AJ, Sherman ME, et al. Human papillomavirus, anal squamous intraepithelial lesions, and human immunodeficiency virus in cohort of gay men. J Infect Dis 1998;178:45-52.

16 Palmer JG, Scholefield JH, Coates PJ, et al. Anal cancer and human papillomaviruses. Dis Colon Rectum 1989;32:1016-1022.

17 Zaki SR, Judd R, Coffield LM, et al. Human papillomavirus infection and anal carcinoma. Retrospective analysis by in situ hybridization and the polymerase chain reaction. Am J Pathol 1992;140:1345-1355.

18 Williams GR, Lu QL, Love SB, et al. Properties of HPVpositive and HPV-negative anal carcinomas. J Pathol 1996;180:378-382.

19 Shroyer KR, Brookes CG, Markham NE, et al. Detection of human papillomavirus in anorectal squamous cell carcinoma. Correlation with basaloid pattern of differentiation. Am J Clin Pathol 1995;104: 299-305.

20 Williams GR, Lu QL, Love SB, et al. Properties of HPVpositive and HPV-negative anal carcinoma. J Pathol 1996;180:378-382.

21 Palefsky J, Holly E, Ralston M, et al. Anal squamous intraepithelial lesions in HIV-positive and HIV-negative homosexual and bisexual men: prevalence and risk factors. J Acquir Immune Defic Syndr Hum Retroviral 1998;17:320-326.

22 Chin-Hong PV, Palefsky JM. Natural history and clinical management of anal human papillomavirus disease in men and women infected with human immunodeficiency virus. Clin Infect Dis 2002;35: 1127-1134.
23 Sun XW, Kuhn L, Ellerbrock TV, et al. Human papillomavirus infection in women infected with the human immunodeficiency virus. $N$ Engl J Med 1997;337:1343-1349.

24 Kiviat N, Rompalo A, Bowden R, et al. Anal human papillomavirus infection among human immunodeficiency virus-seropositive and -seronegative men. J Infect Dis 1990;162:358-361.

25 Williams AB, Darragh TM, Vranizan K, et al. Anal and cervical human papillomavirus infection and irks of anal and cervical epithelial abnormalities in human immunodeficiency virus-infected women. Obstet Gynecol 1994;83:205-211.

26 Sobhan I, Vuagnat A, Walker F, et al. Prevalence of high-grade dysplasia and cancer in the anal canal in human papillomavirus-infected individuals. Gastroenterology 2001;120:857-866.

27 Critchlow CW, Surawicz CM, Holmes KK, et al. Prospective study of high grade anal squamous intraepithelial neoplasia in a cohort of homosexual men: influence of HIV infection, immunosuppression and human papillomavirus infection. AIDS 1995;9: 1255-1262.

28 Caussy D, Goedert JJ, Palefsky J, et al. Interaction of human immunodeficiency and papilloma viruses: association with anal epithelial abnormality in homosexual men. Int J Cancer 1990;162:358-361.

29 Palefsky JM, Holly EA, Ralston ML, et al. Anal squamous intraepithelial lesions in HIV-positive and HIV-negative homosexual and bisexual men: prevalence and risk factors. J Acquir Immune Defic Syndr Hum Retrovirol 1998;17:320-326.

30 Palefsky JM, Holly EA, Hodgeboom CJ, et al. Virologic, immunologic, and clinical parameters in the incidence and progression and anal squamous intraepithelial lesions in HIV-positive and HIV-negative homosexual men. J Acquir Immune Defic Syndr Hum Retroviol 1998;17:314-319.

31 Varnai AD, Bollmann M, Griefingholt H, et al. HPV in anal squamous cell carcinoma and anal intraepithelial neoplasia (AIN). Impact of HPV analysis of anal lesions on diagnosis and prognosis. Int J Colorectal Dis 2006;21:135-142.

32 Castle PE, Fetterman B, Poitras N, et al. Five-year experience of human papillomavirus DNA and Papanicolaou test cotesting. Obstet Gynecol 2009;113: 595-600.

33 Chiao EY, Giordano TP, Palefsky JM, et al. Screening HIV-infected individuals for anal cancer precursor lesions: a systematic review. Clin Infect Dis 2006;43: 223-233. 